\title{
A Kin Selection Paradox
}

By

\section{Mircea Pfleiderer and Jörg Pfleiderer}

(vorgelegt in der Sitzung der math.-nat. Klasse am 17. Juni 2010 durch das w. M. Jörg Pfleiderer)

\begin{abstract}
HAMILTON's [2] famous rule for successful evolution of altruistic behaviour is generalised to include the degree to which an altruistic gene has already spread in the population, and the frequency of altruistic acts (or the population per act). The results are seemingly paradoxical, a main condition for spreading being that altruistic acts should not occur too often.
\end{abstract}

Key words: HAMILTON's rule, relative abundance, altruism, spread of genes, evolution.

\section{Introduction}

One of the starting points of sociobiology was HAMILTON's [2] theory of kin selection. It has often been considered (e.g. [1,5]) as one of the most important achievements in evolutionary biology since DARWIN. Many authors and textbooks even state it to be the only acceptable evolutionary explanation of altruistic behaviour. For our purposes, it is enough to point out that it is generally accepted as a possible explanation.

HAMILTON showed that altruism, despite genetic costs (decrease in reproductive success of the altruist, or in direct fitness - if "fitness" is used in this original but somewhat restricted sense), may evolve if exercising it on a relative confers genetic advantage (increase in the reproductive success of the relative, with corresponding increase in 
the inclusive fitness of the altruist). HAMILTON's number $\mathbf{H}=b r-c$ (where $b$ is benefit to the recipient, $r$ is the relatedness of recipient to donor, and $c$ is cost to donor) should be positive. Since then, many theoretical and empirical papers have dealt with determining $\mathbf{H}$. The debates on how to estimate benefits and costs have not yet come to an end.

By asking for an absolute rather than a relative increase in an altruistic allele, HAMILTON considered (implicitly) the special case of the altruistic allele being very rare as compared to the non-altruistic one. We generalise his calculations by two additions. First, we include the case that the allele in question - the altruistic allele has already spread somewhat in the population. This makes it mandatory to include explicitly the mates of donor and recipient in the calculation. Second, we relate the absolute flow of genes into the next generation to the relative flow by introducing the frequency of altruistic acts, a quantity necessary, in general, for estimating the spread of the altruistic allele.

We restrict ourselves to "true" or "strong" [6] altruistic acts in which both costs and benefits are positive, $c>0, b>0$, and the benefits exceed the costs, the balance being positive, $S=b-c>0$.

Our calculations are simple, straightforward and self-consistent. References to the abundant literature on HAMILTON's rule would not contribute.

\section{The model}

Our model is derived directly from the original model of HAMILTON in order to keep the calculations as similar to HAMILTON's as possible.

We consider a population consisting of two kinds of individuals: those that carry an active altruistic allele - called helper $\mathrm{H}$; by free choice, they may or may not act altruistically - and those in which that allele is not active, i.e., they carry an active non-altruistic allele called non-helper $\mathrm{nH}$; they will never act altruistically. We show in the appendix that this simplified phenotypic description covers the case of diploidy of alleles usual in many instances of observed altruism.

The relative abundance of $\mathrm{H}$ is $q$ with $0<q<1$. A spread of $\mathrm{H}$ in the population is described by an increase in $q$. To estimate such increase as a result of an altruistic act it is necessary to know how often these acts occur. We therefore introduce the number $\alpha$ of altruistic acts in the total population of size $N$, or alternatively the frequency $f=\alpha / N$ of acts, or 
the sub-population of size $M=N / \alpha$ for which - of course, as average one altruistic act occurs.

In our model, costs (c) and benefits (b) are counted in terms of offspring. The altruist or donor, D, loses offspring while the beneficiary or recipient, $\mathrm{R}$, gains offspring. The same situation could, of course, be interpreted differently, for instance, by letting the offspring pay or gain. The lost offspring of $\mathrm{D}$, not born at all or not properly raised, pays by losing its possible fitness wholly or partly. The benefit offspring of $\mathrm{R}$ gains by being additionally born or additionally raised properly and thus obtaining additional fitness. The result, described in detail in the appendix, is, of course, the same.

Diploid offspring gets its genome from two parents, one half from each. In an altruistic act, the donor, D, loses (statistically) a number $c$ of offspring (cost) with the same type $\mathrm{H}$ as the donor, and the same number $c$ with the type of its "mate" (as an abbreviation for the other parent or parents), together a loss of $2 c$. As long as donor and mate are not related (i.e., the mate's probability of being of either type $\mathrm{H}$ or $\mathrm{nH}$ is the same as that of the general population), it is irrelevant whether it is a fixed mate (strict monogamy) or a changing, even unknown mate, in which case the mate need not be mentioned at all. An entirely equivalent description of what happens is that the donor loses $2 c$ offspring to each of which it is related by $r=1 / 2$ and, at the same time, unrelated by $1-r=1 / 2$. Similarly the recipient, R, gains (statistically) a number $b$ (benefit) of offspring with the same type as that of $\mathrm{R}$ itself, plus a number $b$ of the type of its mate, together a gain of $2 b$, to each of which $\mathrm{R}$ is related by $r=1 / 2$ and unrelated by $1-r=1 / 2$.

The essential point is that, for $q \neq 0$, "unrelatedness" is not the same as absence of an altruistic allele. Another interpretation of the same fact is that unrelatedness has vanished; all individuals are related as far as inheriting $\mathrm{H}$ or $\mathrm{nH}$ is concerned, and closer relatedness is increased over the direct relationship $r$. For instance, $\mathrm{D}$ and its offspring are related by an effective relatedness $r^{*}>1 / 2$.

The mates of $\mathrm{D}$ and $\mathrm{R}$ (i.e., the second parents of any offspring of $\mathrm{D}$ and R), considered as unrelated to their partners, may be of either type. Their probability of carrying the altruistic allele influences the offspring's probability of carrying it. Note that this applies not only to the benefit offspring, which is actually born, but also to the cost offspring, which is lost, i.e., not born at all. Without the altruistic act, they would have been born, and they would have had two parents and inherited genes from both, including the genes of the virtual parent. Alternatively, the offspring of $\mathrm{D}$, with genetic components determined by $1 / 2$ relatedness to $\mathrm{D}$ and $1 / 2$ unrelatedness, is just reduced in number. 


\section{HAMILTON's Rule Generalised}

When comparing the outcome of the altruistic act to what would have happened if the altruistic act had not taken place, we find some surprising, even seemingly paradoxical results (details are given in the appendix).

First, a net gain of the altruistic type - as described by HAMILTON's rule in a slightly modified form - does not necessarily mean a spread of the type, i.e., an increase in the relative frequency $q$. The simple reason is that the non-altruistic type may (and will) gain as well. Note that this, of course, means that the population must be growing or, more strictly, growing relatively as a result of the altruistic act. Indeed, the condition $S=b-c>0$ implies that the altruistic act always leads to a population increase as compared to the non-altruistic case where simply $c=b=S=0$.

Second, an altruistic act increases type $\mathrm{nH}$ but not necessarily type $\mathrm{H}$. While an increase in the total amount of the altruistic type (more strictly an allele) depends on conditions (HAMILTON's rule to be fulfilled), the non-altruistic type will in any case increase. This is explained essentially by the fact that the mates of $\mathrm{D}$ and $\mathrm{R}$ (unrelated to $\mathrm{D}$ and $\mathrm{R}$, thus with less probability of being type $\mathrm{H})$ always gain in total $(S>0)$. An equivalent formulation of the same result without mention of mates or second parents is that the unrelated part of the offspring of $\mathrm{D}$ and $\mathrm{R}$ always gains while the related part may not because the related offspring of $\mathrm{R}$ is but partly counted.

Third, the altruistic type can gain more than the non-altruistic one only if the altruistic type has already spread somewhat in the population, i.e., not in those cases HAMILTON considered. The explanation is again that the non-altruistic component of the mates of $\mathrm{D}$ and $\mathrm{R}$ gains too much in total unless $q$ is large enough.

When we now consider the main question of a change in the relative frequency $q$ of alleles, the situation seems to become still more paradoxical. For a relative increase in the altruistic allele, HAMILTON's rule turns out to be a necessary but not a sufficient condition. It needs a further condition, viz., that altruism does not occur too often. The frequency of altruistic acts, $f$, should not be too large, i.e., the population $M$ in which an average of only one act of altruism is to be successfully performed has to surpass a certain minimum size $M_{0}$ (see appendix).

The meaning of this inequality is that there should not be too many helpers: every single helper needs a sufficiently large supporting population of at least $M_{0}$ in which no further helping occurs. A helper helps to increase not only type $\mathrm{H}$ but necessarily also the other type $\mathrm{nH}$. 
As mentioned above, any absolute increase in a type is relatively larger the rarer the type is. The two relevant pairs (donor and recipient, with mates) together have a smaller proportion of type $\mathrm{nH}$ than the rest of the supporting population, which thus must be large enough to decrease the average proportion of type $\mathrm{H}$ and increase the average proportion of type $\mathrm{nH}$ sufficiently.

Another way of looking at the inequality is to consider the reduction to constant population. If it is neutral, i.e., not dependent on whether offspring is produced with help or in spite of help or with no help at all, the reduction of the additional $2 S$ offspring produced by the altruistic act will be taken from the whole population, which has a higher proportion of type $\mathrm{nH}$ than the donor and recipient pairs and their offspring, and thus will favour type $\mathrm{H}$.

Perhaps the most puzzling fact is that an altruistic act that can increase the relative abundance of the altruistic type $\mathrm{H}$ in the general population decreases it in its offspring (the combined offspring of $\mathrm{D}$ and $\mathrm{R}$ plus mates) relative to the parents. These offspring would have been better off in this respect, i.e., had a higher average relative abundance of $\mathrm{H}$, if the altruistic act had not taken place. Such a curious (but, of course, actually quite trivial and, as a fact, even well-known) result can indeed be considered as the underlying reason for the above mentioned paradoxes.

\section{Discussion}

Why can a trivial and well-known fact lead to new results? Simply by considering the effect of an altruistic act not only on the type $\mathrm{H}$ but also on the type $\mathrm{nH}$. This approach is, of course, not new at all. But whoever may have tried it on HAMILTON's problem was definitely not successful in spreading this knowledge.

HAMILTON's rule refers directly only to the special case where the altruistic allele is very rare. In general, it is a necessary but not a sufficient condition for the spreading of an altruistic allele. It seems this restriction has never been noticed in the literature. We have introduced the relative abundance $q$ within the population and - in order to be able to calculate the change in $q$ - the frequency $f$ of altruistic acts, or the average population size $M$ per act. The main result is that altruistic acts should not occur too often, $M>M_{0}$ or $f<f_{0}$.

The limit $M_{0}$ (or $f_{0}$ ) is independent of $q$, which means that the inequality condition can be carried through the evolution from $q \approx 0$ to any final $q$. In other words, the presently observable values of $b, c$ and $M$ 
(population per helper) or $f$ (frequency of helping) might still either obey the inequality, meaning that evolution is still under way with increasing $q$ or has already reached the maximum $q=1$, or obey the equilibrium equality $\left(M=M_{0}\right)$, meaning that the evolution has come to an end (constant $q \leq 1$ ) so that we can actually observe a bygone evolution today.

The fact that $M_{0}$ does not depend on $q$ has a quite interesting consequence. Any gene with an allele that happens to be, for any reason whatsoever, more frequent in altruists than in general will participate in the evolution. Several genes can evolve simultaneously towards the selection of a certain allele. The speed of evolution depends on the individual $q$ and the surplus frequency, $p$, of the allele in question. It is not the same for all genes.

The co-evolution of neutral-to-altruism alleles may have phenotypic consequences that influence the evolution positively (accelerating) or negatively (hindering or even stopping the evolution).

For very small $q$, altruistic acts are apparently rare, and $M$ will easily surpass $M_{0}$. Then HAMILTON's rule is indeed sufficient to guarantee the spreading of the H-gene, rendering the explicit use of the frequency $1 / M$ unnecessary (HAMILTON's case). On the other hand, the end point of the evolution may be twofold. With increasing $q$ and increasing numbers of altruistic acts, $M$ will decrease. One possibility is that $M$ approaches $M_{0}$. The relative abundances of $\mathrm{H}$ and $\mathrm{nH}$ ( $q$ and $1-q$ ) stabilise, and both alleles can survive - a kind of evolutionary stable strategy.

The other possibility is that the helpers "choose" not to be too altruistic and not to help too often so that $M$ always stays larger than $M_{0}$. Then type $\mathrm{H}$ can completely win $(q=1)$, i.e., helpers and non-helpers need not be genetically different. Both behavioural traits are part of the behavioural spectrum of the $\mathrm{H}$ genotype. The choice will presumably depend on environmental circumstances. Considering, for instance, helping in birds (see, e.g., [3]), the two traits, helping and dispersing, fit into slightly different ecological niches: one with less space where the donor can share the territory with the recipient while no new territory is available, and another with more space where a sufficiently large new territory is waiting to be inhabited. For dispersion to occur often enough (that is, new territories not being too rare), the population density is another quantity that should be restricted by evolution.

LEYHAUSEN has pointed out many times (e.g., [4]) that every single animal needs a repertoire of behavioural traits - often competing with and even excluding each other - in order to cope with different situations. The repertoire indeed exceeds the daily needs because this is 
the only way in which the survival rate can be kept sufficiently high in extreme situations. Expressis verbis: Different traits of behaviour may, of course, often derive from different genotypes but equally may not, as seems to be one of the possibilities in our model.

\section{Acknowledgement}

B. TONKIN-LEYHAUSEN kindly cast a critical eye over our English. R. WAGNER and the Journal Club of the Konrad-Lorenz-Institut für Vergleichende Verhaltensforschung, Vienna, were very constructive in helping us to understand the situation. Special thanks are due to H. WINKLER of the same institute.

\section{References}

[1] Alonso, W. J., SchUCK-PAIM, C. (2002) Sex ratio conflicts, kin selection, and the evolution of altruism. Proc. Natl. Acad. Sci. USA 99: 6843-6847

[2] Hamilton, W. D. (1964) The genetical evolution of social behaviour. J. Theor. Biol. 7: 1-52

[3] Grafen, A. (1984) Natural selection, group selection, and kin selection. In: Krebs, J. R., Davies, N. B. (eds.) Behavioural ecology, an evolutionary approach, 2nd edn, pp. 63-84. Blackwell Scientific, London

[4] Leyhausen, P. (1973) On the function of the relative hierarchy of moods. In: LORENZ, K., LEYHAUSEN, P. (eds.) Motivation of human and animal behavior, pp. 144-247. Van Nostrand, New York

[5] Trivers, R. (2000) William Donald HAMILTON (1936-2000): obituary. Nature 404: 828

[6] WILSON, D. S. (1977) Structured demes and trait-group variation. Amer. Nat. 113: $606-610$

Authors' addresses: Prof. Dr. Jörg Pfleiderer, Dr. Mircea Pfleiderer, Institut für Astround Teilchenphysik der Leopold-Franzens-Universität Innsbruck, Technikerstraße 25, 6020 Innsbruck, Österreich; Karoo Cat Research, Honingkrantz, P.O. Box 20, Fish River ZA-5883, South Africa. E-Mail: felis@ isat.co.za; joerg.pfleiderer@uibk.ac.at.

\section{Appendix}

\section{Assumptions of the Calculation}

Consider an (arbitrarily large) population of $N$ individuals in which altruism occurs. The average altruistic donor $\mathrm{D}$ pays the cost $2 c$ in offspring while the recipient $\mathrm{R}$ gains the benefit $2 b$ in offspring. Let $\alpha$ be the number of altrustic acts within the population. Then $M=N / \alpha$ is the population per altruistic act, and $f=1 / M=\alpha / N$ is the frequency of altruistic acts, or the number of acts per individual. These numbers should, of course, be considered as statistical averages, as "the" altruistic act can only be a statistical act. 
We approximate the altruistic genetics by a single gene consisting of at least two alleles, an "altrustic" one, a, and an opponent, $\mathbf{A}$, that includes the sum of all other existing alleles, whether just one or more. There are 3 genotypes: aa, $\mathbf{a A}$, and $\mathbf{A A}$. The relative abundance of $\mathbf{a}$ in the total population or the probability (= abbreviated $\mathrm{P}$ ) of carrying $\mathbf{a}$ is $\mathrm{P}(\mathbf{a})=q$, that of $\mathbf{A}$ is $\mathrm{P}(\mathbf{A})=1-q$, with $0<q<1$. That is, the total number of allele $\mathbf{a}$ in the (diploid) population is $m(\mathbf{a})=2 q N$, or an averaged number of $2 q$ per individual, while that of $\mathbf{A}$ is $m(\mathbf{A})=2 N(1-q)$. Actual altruists are supposed to have, on average, a different, higher, relative abundance of $\mathbf{a}$, which we call $\mathrm{P}(\mathbf{a})=p$ (with $p>q$ and a total average number $2 p$ of a-alleles per individual), and a correspondingly smaller relative abundance $\mathrm{P}(\mathbf{A})=1-p<1-q$ of A. Spreading of altruism in the population is thus described by an increase in $q$.

It is not necessary to specify which and how many of the a-carriers are possible altruists. An allele can reasonably be called altruistic only if it is present at least once (heterozygote) in any altruist, which implies $p \geq 1 / 2$. The following calculation does not, however, depend on this assumption. The same calculation holds for any other allele that is connected with altruism, not in the sense of causing it, as the allele $\mathbf{a}$ is supposed to, but only in the sense of being more frequent in altruists than in the general population. More generally, a whole group of alleles that happen to be more frequent in actual altruists than in others can thus evolve simultaneously.

When we compare, as HAMILTON did, the statistical results of altruistic acts with the results expected if the acts had not taken place, i.e., if the altruists had chosen not to behave altruistically, the first fact to notice is that unrelatedness does not mean that allele $\mathbf{a}$ is not present at all but rather that it is present with a probability $q^{\prime}<q$ as defined below.

The actual altruist or donor D has, as mentioned, the free choice of helping or not helping a recipient $\mathrm{R}$ which is its relative by relationship $r$, defined as usual (siblings or children $r=1 / 2$, grandchildren $r=1 / 4$, etc.). R contains allele a with the probability $\mathrm{P}(\mathbf{a})=p^{\prime}$, consisting of two parts. It derives, with probability $r$, from the relatedness to $\mathrm{D}$ with $\mathrm{P}(\mathbf{a})=p$, and, with probability $1-r$, from ancestors unrelated to $\mathrm{D}$, with $\mathrm{P}(\mathbf{a})=q^{\prime}$. That is, each allele of $\mathrm{R}$ coinciding with an allele in $\mathrm{D}$ is inherited either from a common ancestor (relationship $r$ ) or from a different ancestor carrying the same allele but not passing it on to D while passing it on to R (so to speak "un-relatedness" $1-r$ ). Together, the probability of $\mathrm{R}$ carrying allele $\mathbf{a}$ is

$$
p^{\prime}=r p+(1-r) q^{\prime} .
$$

Here, $q^{\prime}$ is the reduced proportion of allele $\mathbf{a}$ in the rest of the population $N$ without all the donors and recipients $\mathrm{D}$ and $\mathrm{R}$ (as compared to $q$ as proportion of the whole population), deriving from the fact that $\mathrm{D}$ and $\mathrm{R}$ carry an increased proportion of $\mathbf{a}$. In detail,

$$
\begin{aligned}
& N q=(N-2 \alpha) q^{\prime}+\alpha\left(p+p^{\prime}\right), \text { or } \\
& q^{\prime}=(M q-p-p r) /(M-1-r)<q, \text { thus } \\
& q^{\prime}<q<p^{\prime}<p .
\end{aligned}
$$

As mentioned in the text, one can define the relatedness between $\mathrm{D}$ and $\mathrm{R}$ by a direct relatedness $r$ (with unrelatedness $q^{\prime}$ ) or by an effective relatedness $r^{*}=r+(1-r) q^{\prime} / p$.

The next fact to notice is that the population size $N$ necessarily changes, benefit minus cost being positive in the case of "true" altruism. The reduction to constant population size within the next or later generations is secondary and of no relevance 
here, as long as it is neutral, i.e., affecting all individuals equally in a statistical sense, not depending on whether or not they originate from an altruistic act. Accordingly, we ask for the change, as a result of the altruistic acts, in $N$, in $m$ (a) (this is HAMILTON's question), in $m(\mathbf{A})$, and in $q$, as compared to the outcome of no altruistic acts at all.

Both $\mathrm{D}$ and $\mathrm{R}$ are supposed to have unrelated partners with the probability $q^{\prime}$ of also carrying a. As mentioned in the text, the partners of $\mathrm{D}$ and $\mathrm{R}$ may be one each, or many. They are meant as statistical partners (this is exactly the same as forgetting about the partners but introducing instead a corresponding unrelatedness), all with the same probability $q^{\prime}$ and thus indistinguishable. The combined ( $\mathrm{D}+\mathrm{R}$ plus partners) relative abundance of $\mathbf{a}$ is

$$
q^{*}=\left(p+p^{\prime}+2 q^{\prime}\right) / 4 .
$$

This is, of course, the same as the outcome of considering only the relevant parents ( $p$ and $p^{\prime}$, relatedness to offspring $r={ }^{1} / 2$ ) and the unrelated general population with $q^{\prime}$ (unrelatedness $1-r=1 / 2$ ).

While it is generally not a particular problem to settle on a specific donor, the identity of the recipient might well be a matter of opinion. For instance, if a young female bird helps her mother raise next year's offspring, who benefits, mother or offspring? Both opinions yield the same result. The benefit consists of additional offspring. In the first case, the relatedness of donor $(p)$ to recipient $\left(p^{\prime}\right)$, i.e. helping daughter to mother, is $r=1 / 2$, thus $p^{\prime}=1 / 2\left(p+q^{\prime}\right)$. Relatedness of the beneficiary to benefit is also $1 / 2$, thus $\mathrm{P}(\mathbf{a})=1 / 2\left(p^{\prime}+q^{\prime}\right)=1 / 4 p+3 / 4 q^{\prime}$. In the second case, the relatedness of donor to half-siblings ((beneficiaries) is $r=1 / 4$, while the beneficiaries are identical with the benefit $(r=1)$, thus $\mathrm{P}(\mathbf{a})=1 / 4 p+(1-1 / 4) q^{\prime}$.

Generally, the situation can be described by one or two or many beneficiaries as well as donors. Costs and benefits may be counted by offspring, or by what happens in the following generation, or otherwise. Appropriately formulated, the results will be the same. But it should be remembered that, in the formulation of our model, the mates of donor and recipient (one mate, several mates, virtual mate) do share costs and benefits.

\section{Comparison of Altruistic vs. Non-altruistic Act}

We can now compare the two possibilities of no help (ordinary procedure) and help (altruistic procedure).

First case: No altruistic act, no benefit and no cost. Both types have the same average number of offspring $n$ per animal, or $2 n$ per pair. The total number of offspring is $N n$. Constant population (same number of offspring as number of parents) corresponds to $n=1$. The relative abundance of allele a stays constant at the value $q$. In particular, the relative abundance of the combined offspring of each quartet $D+R+$ mates (or $1 / 2$ $(\mathrm{D}+\mathrm{R})$ plus $1 / 2$ unrelatedness $)$ is

$$
n\left(p+p^{\prime}+2 q^{\prime}\right) / 4 n=q^{*}
$$

which is the same as that of the averaged parents. This result is, of course, a (rather trivial) expression of the HARDY-WEINBERG rule.

Second case: Altruistic act with benefit and cost. The (average) altruistic animal D and its partner (so to speak a virtual partner as regards the loss) produce (of course as average) $2(n-c)$ offspring, i.e., $2 c$ less offspring (cost); $\mathrm{R}$ and its partner produce 2 $(n+b)$ offspring, a benefit of $2 b$. The total number of offspring is $n N+2 \alpha(b-c)$. 
Constant population corresponds to $n=(M-2 S) / M$, which for true altruism $(S>0)$ is $<1$. The relative abundance of $\mathbf{a}$ in the combined offspring of $\mathrm{D}+\mathrm{R}+$ mates (equivalent, as above, to $1 / 2(\mathrm{D}+\mathrm{R})$ plus $1 / 2$ unrelatedness) is

$$
\begin{aligned}
q^{\prime \prime} & =\left((n-c)\left(p+q^{\prime}\right)+(n+b)\left(p^{\prime}+q^{\prime}\right)\right) /(4 n+2 S) \\
& =q^{*}-\frac{1}{4}\left(p-p^{\prime}\right)(b+c) /(2 n+S)<q^{*} .
\end{aligned}
$$

The surprising and seemingly paradoxical (while, as mentioned, actually trivial) result $q^{\prime \prime}<q^{*}$ is that from which all the other seemingly controversial results can be understood: the altruistic act that (as HAMILTON showed and we are only verifying under more general conditions) can increase the relative abundance of an altruistic allele (and thus an altruistic genotype) in the general population will always decrease this abundance in its offspring, as compared to the parents. These offspring have a smaller relative abundance of the a allele than they would have had without the altruistic act.

The "altruistic" balance (second case minus first case) for the total offspring of the (average) sub-population of size $M=N / \alpha$ in which one altruistic act occurs, i.e., the balance per average altruistic act, is

$$
D_{0}=\{n M+2(b-c)\}-n M=2 S>0,
$$

showing that the true altruistic act always produces a population increase as compared to the non-altruistic case. The balance per act of allele $\mathbf{a}$ is

$$
\begin{aligned}
D_{1} & =2\left\{n M q+b\left(p^{\prime}+q^{\prime}\right)-c\left(p+q^{\prime}\right)\right\}-2 n M q \\
& =2\left\{q^{\prime}(b(2-r)-c)+p(b r-c)\right\}=2\left\{p \mathbf{H}+t q^{\prime}\right\}
\end{aligned}
$$

(with $t:=b(2-r)-c>0$ and $\mathbf{H}:=b r-c$ ) while the balance per act of the other allele A is

$$
D_{2}=2\left\{b\left(1-p^{\prime}+1-q^{\prime}\right)-c\left(1-p+1-q^{\prime}\right)\right\}=4 S-2\left\{p \mathbf{H}-t q^{\prime}\right\}>0 .
$$

Trivially, the balance of the total number of alleles, $D_{1}+D_{2}$, equals twice the difference in individuals, $D_{0}$.

What do these numbers tell us?

First, we ask HAMILTON's question. The total number of allele a increases if $D_{1}>0$. As is to be expected, we find the famous rule $\mathbf{H}>0$ of HAMILTON, but it is relaxed as soon as the allele has already spread in the population, $q^{\prime}>0$. This does not necessarily mean that it spreads further, because, in a growing population (here by $\alpha D_{0}$ ), all alleles may increase in absolute number.

Second, in a true altruistic case $(S>0), D_{2}$ is always positive (this follows from the fact that $\left.q^{\prime}<p^{\prime}<p\right)$. The $\mathbf{A}$ allele cannot lose.

Third, allele a increases more than the other allele $\mathbf{A}\left(D_{1}>D_{2}\right)$ only if

$$
q^{\prime}>(S-p \mathbf{H}) / t>0,
$$

i.e., only if allele a has already spread sufficiently in the population for the unrelated partners (second parent) to have a large enough probability of also carrying allele a.

Fourth, the real question is, of course, whether the relative abundance $q$ of a increases,

$$
d q / d t>0 \text {. }
$$


We find that $q^{\#}$ of the offspring, given by the ratio of the absolute number of (statistically expected) a-alleles in the offspring,

$$
m^{\#}(\mathbf{a})=2 N q n+2 \alpha b\left(p^{\prime}+q^{\prime}\right)-2 \alpha c\left(p+q^{\prime}\right),
$$

and the total number of alleles in the expected offspring,

$$
2 N^{\#}=2 N n+4 \alpha S,
$$

that is,

$$
q^{\#}=m^{\#}(\mathbf{a}) / 2 N^{\#}=q+(p-q)\{M \mathbf{H}-2 S(1+r)\} / 2\left(N^{\#} / \alpha\right)(M-1-r),
$$

is larger than $q$ of the original population $N, q^{\#}>q$, only if, as long as $q<1$, the nominator bracket \{\} is positive. In the altruistic case $S>0$, HAMILTON's rule $\mathbf{H}=b r-c>0$ turns out to be a necessary condition but not a sufficient one. There is a further condition,

$$
\begin{array}{rlrl}
M>M_{0} & \text { with } & M_{0}=2 S(1+r) / \mathbf{H}, & \text { or } \\
f<f_{0} & \text { with } & f_{0}=\mathbf{H} /(2 S(1+r)), & \text { or } \\
\alpha<\alpha_{0} & \text { with } & \alpha_{0}=N f_{0} .
\end{array}
$$

This does not depend on $q$, so loss and benefit need not adapt during evolution. As mentioned above, several genes may simultaneously evolve towards a preferred allele. They may, however, have different $q$ s and $p$ s.

The limiting case $M=M_{0}$ means that there is no gain of either type. Equilibrium can in principle be reached at any value of $q$. An inequality $M>M_{0}$ would probably indicate that the present end point of evolution is $q=1$. The third possible case, $M<M_{0}$, would perhaps indicate a present decrease of $q$ or an at present unstable situation with $q=1$. 